\title{
Research on breakdown of high pressure start of CPIGENIII HPA
}

\author{
Liu Shuai, Chen Xi, Yao Xuelu
}

China Satellite Maritime Tracking and Control Department, Jiangyin, 214431, China

Key words: CPI; high power amplifier; high voltage start; control protection

\begin{abstract}
CPIGEN III high power amplifier in the high pressure after the emergence of high pressure, causing high pressure start fault alarm. In order to improve the level of emergency response of the transmitter, this paper first combs the sensor type and signal form of the high-voltage starter, and then analyzes the circuit principle and control logic of the high-voltage start-up. Finally, the fault inducement is analyzed and the high-voltage start fault .
\end{abstract}

\section{Introduction}

I station C-band transmitter using the US CPI company GEN III 3kW klystron high power amplifier, with stable, high reliability, but the internal strength of the amplifier structure is very complex, high-power, high voltage, high current devices and IC more, more difficult to maintain. In a power process, the high power amplifier appears out of high voltage phenomenon, the panel prompts "STRATER OR AC FAULT" warning instructions, post staff on the phenomenon of this failure related analysis.

\section{High-voltage start-up related to the type of sensor and signal form}

\subsection{High-voltage start-up status sensor type}

High voltage start is controlled by the CONTROLLER CCA (control logic unit) control signal to control the AC contactor pull, and then return to the state by a return to determine the AC contactor to pull the state, if the return signal is abnormal, the panel indicates "STRATER OR AC FAULT Alarm information. The entire high-pressure status indication circuit, and its associated sensors are: (1) RKS-11DX-12 relay: INTERLOCK CCA (interlock unit) on the K1 and K2; (2) MICRO SWITCH micro switch: AC contactor with the micro switch. One relay is to control the role of $110 \mathrm{~V}$ power on and off, the role of micro-switch is to return to the state of the AC contactor pull. Figure 1 on the left for the Furnas AC contactor on the micro switch, the right for the 14-C-120A AC contactor on the micro-switch, are pin-type, US manufacturing, life can be more than $800 \mathrm{~W}$ times.

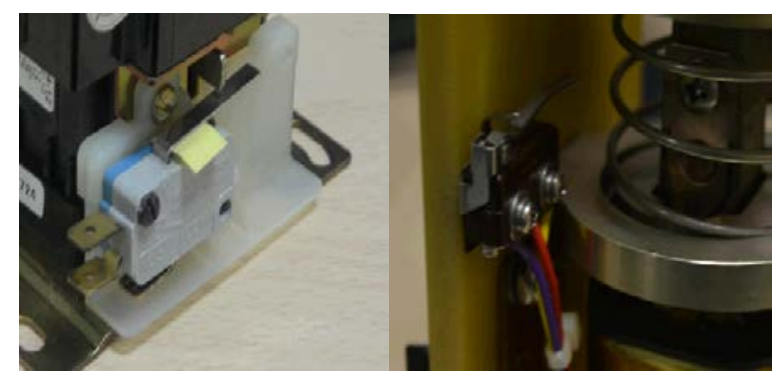

Figure 1. Micro Switch

\subsection{Signal form analysis}

There are two types of signals related to the sensor: (1) Control signal: When the "Transmit" button on the panel is pressed, the high voltage command is sent to the CONTROLLER CCA via the panel, and the HV ENABLE Enable) signal, the enable level is $15 \mathrm{~V}$ (J2 of the 4 core), relay K1 open; when the panel on the high-voltage operation, the enable level is pulled down to $0 \mathrm{~V}$, relay $\mathrm{K} 1$, J1 1, 3 core pass, $110 \mathrm{~V}$ high voltage control sent to the high voltage starter; (2) state return signal, high voltage start circuit in the last Furnas AC contactor after the suction, press the pin to press the 
micro switch, micro switch NO3 Port and COM port conduction. CONTROLLER CCA J9 port through the acquisition of micro-switch connection NO3 and COM port status signal to determine whether the normal high-voltage start. Micro switch is not pulled, NO3 port level is $15 \mathrm{~V}$, COM port ground, when the pull-in, NO3 port level is pulled down to 0V. If NO3 is not pulled low, the panel indicates "STRATER OR AC FAULT".

\section{Analysis of High Voltage Startup Protection Logic and High Voltage Startup Circuit}

\subsection{High-voltage start-up control protection logic}

The $110 \mathrm{~V}$ control voltage is supplied to the switch panel by the A2J3 adapter after the 110L control voltage is supplied by the transformer, and after the INTERLOCK CCA is connected to the AC contactor K1 (No. 01020530 model 14-C-120A) of the high voltage assembly. AC contactor K1 (model number 42BE35AF560R) H pin, while jumper to K1 with micro switch NO pin, K1 pull, $110 \mathrm{~V}$ voltage through the $\mathrm{K} 1 \mathrm{micro}$ switch COM pin to K3 (model 49MD06FA) delay (In order to make K1 closed earlier than K2, for the protection circuit) and then sent to the switch panel AC contactor K2 (model 42BE35AF560R) H feet, K2 pull, the attached micro switch closed, and CONTROLLER CCA module J9 (5-pin: SYS COM32 core: step start complete;) connected to the state of the feedback signal line between the two conduction, so that the detection level from $15 \mathrm{~V}$ was pulled down to $0 \mathrm{~V}$, that is, high-pressure control normal closed-loop.

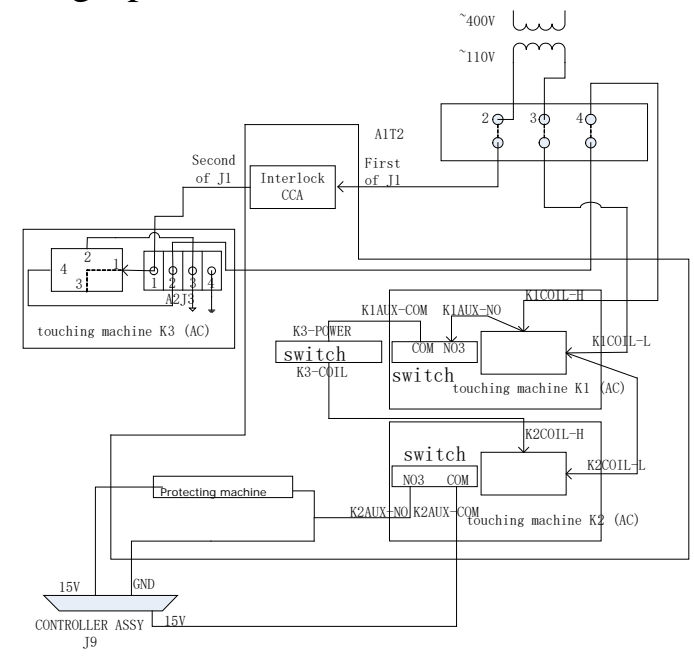

Figure 2. High voltage start control logic diagram

\subsection{Analysis of High Voltage Starting Circuit}

380V from the power distribution box to the TB1 switch row, the MAIN POWER open space, respectively, COOLING open space and BEAM P / S empty open power supply, which entered a high-voltage components of the $380 \mathrm{~V}$ need to go through in parallel with the K1, K2, The two AC contactor by the $110 \mathrm{~V}$ control, only the HV ENABLE to send out, $110 \mathrm{~V}$ voltage into the control loop, to be $380 \mathrm{~V}$ after $\mathrm{K} 2$ pull into the voltage to a high-voltage components to transform the same time, 380V electric shunt to REGULATOR The secondary high voltage module is used to boost the $6 \mathrm{kV}$ DC output from the primary high voltage module to $8 \mathrm{kV}$. Figure 3 shows, once the high-voltage control circuit problems will make the door switch panel AC contactors K1 and K2 can not pull, resulting in $380 \mathrm{~V}$ can not be sent to a high-voltage components and generate high-voltage start fault alarm. 


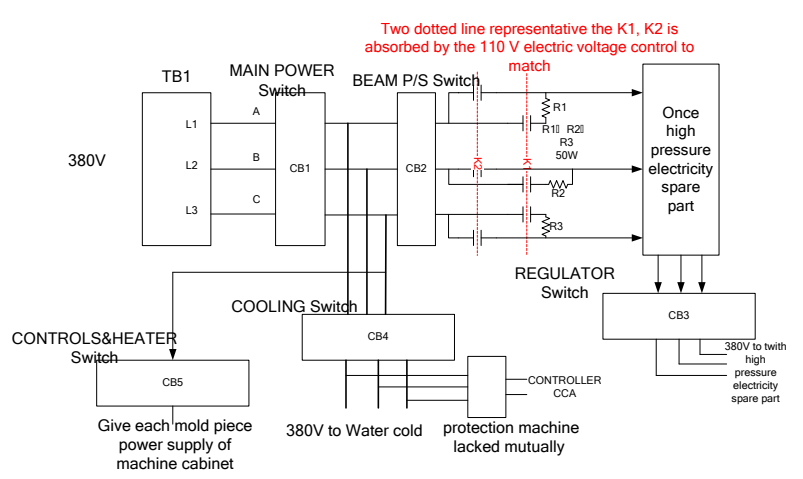

Figure 3. High - voltage start - up circuit

\section{Cause Analysis of Transmitter High Voltage Starting Failure}

Through the above analysis of the circuit principle and the control program, we can see that the incentive for the high voltage start fault of the transmitter lies in the following five aspects: a). 110V transformer output is abnormal; b). CONTROLLER CCA enable instruction or acquisition judgment is wrong; c). INTERLOCK CCA on the relay $\mathrm{K} 1$ and $\mathrm{K} 2$ failure caused $110 \mathrm{~V}$ control voltage is not sent to the high voltage start circuit; d). A high-voltage components of the AC contactor $\mathrm{K} 1$ and its accompanying micro-switch pull-in failure caused $110 \mathrm{~V}$ control voltage can not be sent to the next AC contactor; e). The delay relay on the door switch panel or the AC contactor K1, K2 and its associated micro switch malfunction.

To locate the fault location, perform the following troubleshooting steps:

a). Measuring $110 \mathrm{~V}$ transformer output

With a multimeter to measure the adapter plate A1T2 2,3 pin (transformer output), the measured result is $110 \mathrm{~V}$, that the transformer output is normal.

b). Replace INTERLOCK CCA

Replace the interlock unit, fault recurrence, eliminate the impact of the relay board.

c). And shields the AC contactor $\mathrm{K} 1$ in the primary high voltage module

Will be a high-voltage components within the AC contactor $\mathrm{K} 1$ of the micro-switch shielding, so that it is normally closed state, plus high pressure after the fault recurrence, excluding a high-voltage components within the impact of AC contactor K1.

d). Replace the AC contactor on the door switch panel

Replacement of AC contactor K2 spare parts, several times on the next high-voltage operation, fault phenomenon is not complex, problem positioning.

After analysis, CONTROLLER CCA by detecting AC contactor K2 micro switch with the closed state to determine the high-voltage start-up anomalies. The AC contactor K2 due to internal dust caused by its pin is not in place, so micro-switch closed lax, resulting in abnormal feedback signal, resulting in high-pressure start alarm and automatic high-pressure phenomenon. The NO3 port of the micro switch is disconnected from the COM port and the level of the NO3 port is not pulled low, the control logic unit judges that the high voltage starting abnormality is caused and the control logic unit turns off the high voltage enable signal, $110 \mathrm{~V}$ control signal can not be sent to the circuit, all the AC contactors in the loop pop up, resulting in $380 \mathrm{~V}$ can not be sent to K2 through a high-voltage components transformer, this time panel issued an alarm indication "STRATER OR AC FAULT".

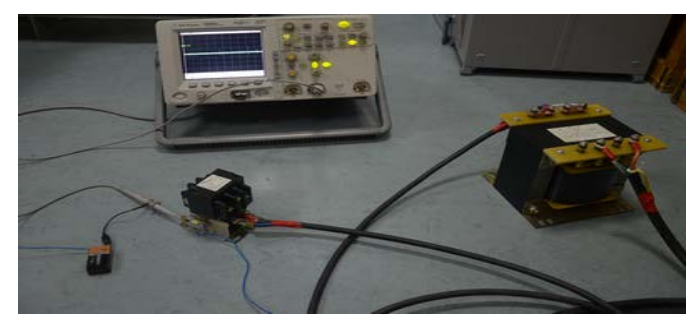

Figure 4. AC contactor machine under test platform

On the replacement of the K2 AC contactor for inspection, check the contactor contacts found 
normal, no oxidation or adhesion, suction coil cavity slightly dust, dust maintenance and re-assembly, and under the machine to build as shown in Figure 4 The test platform tests the AC contactor K2, and the oscilloscope observes the micro switch output voltage to be stable. The exchange of contactor re-installed for more than 50 hours of high-voltage pager test, the fault is not reproduced, the equipment is working properly.

\section{Summary}

This paper describes the CPIGEN III high power amplifier high voltage start control logic, pointed out that may cause high voltage start fault problems, and finally the fault location in the AC contactor dust lead to high pressure start failure. Although the use of water-cooled cooling tube, but the cabinet is still used within the air cooling to exclude other equipment heat, a lot of air does not stay easy to make dust in the high-pressure sensitive devices within the accumulation, resulting in serious failure such as dropping high pressure. Follow-up staff will regularly on the amplifier exhaust fan and air filter for dust, while the use of isopropyl alcohol cleaning high-voltage devices to ensure that the equipment operating environment is good.

\section{Reference:}

[1] Yu Bing, Gao Zumin. CPIGENIV3kW klystron high power amplifier typical fault analysis and processing. No. 11. 2010. Broadcast and television technology

[2] An Ding. GENIV Summary of experience in running high power amplifier. No. 6. 2008. Broadcasting and Television Technology 